with in-hospital mortality (odds ratio $1.53,95 \%$ confidence interval [CI] $0.37,6.29 ; \mathrm{p}=0.55$ ). There was no difference in discharge disposition or time to hospital readmission by corticosteroid treatment. There was a possible increase in time to death following discharge in patients receiving corticosteroids (Figure). DISCUSSION/ SIGNIFICANCE OF IMPACT: Conclusions: This study suggests that treatment of acute exacerbations of interstitial lung disease with corticosteroids does not improve short-term outcomes, including in-hospital mortality, all-cause non-elective re-hospitalization or death within 6 months of discharge. Further research in larger cohorts is needed to more definitively assess this relationship.

3371

\section{The Devil is in the Details: Unbalanced Gains in Healthcare Access and Affordability in the Health Insurance Exchanges}

Uriel Kim ${ }^{1}$, Johnie Rose and Siran Koroukian

${ }^{1}$ Case Western Reserve University

OBJECTIVES/SPECIFIC AIMS: Evaluate how access and affordability has changed before and after the implementation HIEs in three subpopulations. The subpopulations are individuals who are currently insured through the HIE but were previously: 1 . Insured through Employment-based insurance (PEBI subpopulation) 2. Insured through Private Insurance (PPI subpopulation) and 3. Uninsured (PU subpopulation). The three access and affordability measures are: Outcome measure 1 . Did not fill a prescription in the past year due to cost Outcome measure 2. Could not get needed medical exam in the past year due to cost and Outcome measure 3. Had problems paying medical bills in the past year. METHODS/ STUDY POPULATION: We analyzed the de-identified public use data from the 2012 and 2015 Ohio Medicaid Assessment Survey (OMAS). Sponsored by the Ohio Department of Medicaid, Ohio Department of Health, and the Ohio State University, the OMAS is a representative cross-sectional survey of non-institutionalized Ohio residents, regardless of their Medicaid status. In order to "longitudinalize" the 2012 and 2015 cross-sectional data of the OMAS, we employed a propensity score-based approach. We started with the 2015 OMAS, and carefully characterized each of the PEBI, PPI, and PU subpopulations along 17 demographic, health utilization, health behavior, and health status covariates using a propensity score model. Then, we identified controls for the three subpopulations within the 2012 OMAS data using the propensity scores. Finally, we estimated the odds ratios for the three outcome measures between 2012 and 2015. RESULTS/ANTICIPATED RESULTS: In 2015 there were approximately 201,381 adults (unweighted count =996) who were insured through the HIE in Ohio. Of those individuals, $17.7 \%$ fell into the PEBI subpopulation, $17.6 \%$ fell into the PPI subpopulation, and $53.3 \%$ fell into the PU subpopulation; the balance of the respondents (11.4\%) reported previously having Medicaid, or "Other" insurance. There are several key differences in the covariates at baseline between the three subpopulations. In general, the PU subpopulation tended to younger, more minority, more socioeconomically disadvantaged, and more likely to not have a primary care provider compared to both the PEBI and PPI subpopulations. In the 2012 data, we were able to identify 170 controls for the PEBI subpopulation, 167 controls for the PPI subpopulation, and 516 controls for the PU subpopulation. Compared to 2012, in 2016 (after the implementation of the HIEs):. Outcome measure 1: The PEBI subpopulation was more likely to report not filling a prescription in the past year due to cost (there were no significant changes in the PPI or PU subpopulations). Outcome measure 2: The PEBI subpopulation was more likely to report not getting a needed medical exam or medical supplies in the past year due to cost. The PPI subpopulation was less likely to report not getting a needed medical exam or medical supplies in the past year due to cost. There were no significant changes for the PU subpopulation for this outcome measure. Outcome measure 3: There were no changes in the "had problems paying medical bill in the past year" outcome across the three subpopulations. DISCUSSION/SIGNIFICANCE OF IMPACT: This is among the most detailed studies of health insurance exchanges known to the investigators. Analyzing outcomes at the subpopulation level illustrates that there have been unbalanced gains in access and affordability as a result of the HIEs. In general, those who were previously insured through employer-based insurance saw their access and affordability decrease; those previously insured through private insurance saw modest increases to access and affordability; and perhaps most surprising, those that were previously uninsured saw no changes to their access and affordability. Future studies will incorporate 2017 OMAS data (when it becomes available) to see if these trends persist over time. During this time of rapid health systems and health policy change, our study adds an important contribution to the discussion surrounding how to best deliver highly effective and efficient health care.

3232

\section{Translational Science 2019}

Paul C Adjei ${ }^{1}$, Michael R. Jordan, Jennifer Chow and Janis Breeze ${ }^{1}$ Tufts Medical Center

OBJECTIVES/SPECIFIC AIMS: We hypothesize that VL testing varies by geographic sub-region, country, age, gender, mode of transmission, year of diagnosis, and country of origin; and also that a higher prevalence of VL testing may be associated with higher prevalence of population-level VL suppression. Our primary aim is to determine country- and regional-level factors that are associated with viral load testing amongst HIV patients. Our secondary aim is to explore the association between prevalence of viral load testing and viral load suppression at the population level. METHODS/ STUDY POPULATION: This is a retrospective analysis of deidentified individual-level data reported to the European Surveillance System (TESSy). The TESSy is a database of communicable diseases (including HIV) for the ECDC and WHO European Regional Office. It captures data from 31 European Union/European Economic Area (EU/EEA) countries and 23 non-EU/EEA countries. Stored data is from year 2000. TESSy is used for data analysis and production of outputs for public health action. The patient cohort include adults older 18 years, whose last clinic attendance was reported in 2014 or later, or whose viral load test was reported in the year of the visit or the year before the year of their last reported clinic attendance. Patient demographic data include age, sex, mode of transmission, country of origin (migrants), country of diagnosis, geographic region, last clinic attendance, viral load and therapy status. Geographic region will be categorized into East, West and Centre as per WHO guidelines. Countries will be categorized and analyzed according to their European Union (EU)-, European Economic Area (EEA)- and income (GDP)-status, using current World Bank and International Monetary Fund (IMF) guidelines. All statistical analysis will be performed in R-Studio and $\mathrm{R}$ i386 3.0.2. Missing data will be characterized in terms of quantity (how much is missing) and pattern (random versus non-random) and impact on covariates to be tested. Multiple data imputations would be used in cases where 